\title{
A COMPARISON OF PHYCOCYANINS FROM THREE DIFFERENT SPECIES OF CYANOBACTERIA EMPLOYING RESONANCE-ENHANCED COHERENT ANTI-STOKES RAMAN SPECTROSCOPY
}

\author{
S. SChneider ${ }^{* 1}$, C.-J. Prenzel ${ }^{1}$, G. BrehM ${ }^{1}$, P. Gedeck ${ }^{1}$, P. S. Maruthi Sai ${ }^{1,2}$, \\ L. GOTTSCHALK ${ }^{3}$ and H. SCHEER ${ }^{3}$
}

Institut für Physikalische und Theoretische Chemie, Univ. Erlangen, Egerlandstr. 3, W-8520 Erlangen, Germany, ${ }^{2}$ Department of Biochemistry, University of Delhi, South Campus, Benito Juarez Road, New Delhi 110021 , India, and ${ }^{3}$ Botanisches Institut der Ludwig-Maximilians-Universität, Menzingerstr. 67, W-8000 Müchen 19, Germany

(Received 6 April 1992; Accepted 13 July 1992)

\begin{abstract}
Resonance-enhanced coherent anti-Stokes Raman spectra are recorded for monomers and trimers of phycocyanin from three different cyanobacteria: Westiellopsis prolifica, Mastigocladus laminosus and Spirulina platensis. It is shown that upon aggregation from monomer to trimer the electronic structures of both the $\alpha 84$ and $\beta 84$ chromophores are changed. The spectra of the trimers originating from $S$. platensis and $M$. laminosus are very similar to each other, but distinctly different from the spectrum of $W$. prolifica.
\end{abstract}

\section{INTRODUCTION}

Cyanobacteria possess brilliantly colored phycobiliproteins in supramolecular aggregates called phycobilisomes (PBS) ${ }^{1}+$ Their function is to absorb light in the spectral region between 500 and $650 \mathrm{~nm}$ and to transfer the excitation energy to the photosynthetic reaction centers ${ }^{2}$ Phycocyanin (PC), which is found within the rods of the antenna complex, is present in all species of cyanobacteria studied so far. The chromophores of PC, designated as phycocyanobilins (PCB), are open chain tetrapyrrole systems attached covalently to a cysteine residue in each of the two polypeptide chains called the $\alpha$ - and $\beta$-subunit, respectively. ${ }^{3}$ From high-resolution X-ray crystallography it is known that the three distinct tetrapyrrole chromophores of the monomer $(\alpha 84, \beta 84$ and $\beta 155)$ are held in very similar, extended, but nonplanar geometries by interaction with the surrounding protein. ${ }^{4}$ As theoretical studies demonstrate, the chromophore-protein interactions are important not only for the rigid geometry of the chromophores, but also for the fine-tuning of the absorption characteristics of the different chromophores. ${ }^{5}$

Phycocyanins isolated from different organisms exhibit rather similar absorption spectra and fluorescence properties and show characteristic changes in absorption upon aggregation from the monomeric to the trimeric state. One of the open questions in the attempt to understand spectral properties and energy transfer within the phycobilisomes and their constituent biliproteins is, therefore, whether the chromophore geometry is the same in different biliproteins and whether there is a change of chromophore geometry upon aggregation. Spectroscopic techniques, like Raman spectroscopy, give, even for solutions, structure-specific information in the form of vibrational frequencies and band intensities. A correlation between these quantities and molecular struc-

*To whom correspondence should be addressed.

† Abbreviations: CARS, coherent anti-Stokes Raman scattering; PBS, phycobilisome; PC, phycocyanin; PCB, phycocyanobilin. ture is, however, complex and will not be possible within the near future. By comparison of spectra of different biliproteins it is, however, possible to decide on the question, whether the molecular structure is different when going from one biliprotein to the other or when changing the state of aggregation.

In the past, both spontaneous resonance Raman spectroscopy and coherent anti-Stokes Raman scattering (CARS) have been applied successfully to study biliproteins. ${ }^{\sigma-9}$ Both techniques have advantages and disadvantages. One of the advantages of CARS is that fluorescence is no problem even when the excitation wavelength coincides with the longwavelength transition. Due to the differences in the absorption maxima of the three chromophores, a partial separation of the signal contributions of each chromophore is achieved by selective resonance enhancement. In this contribution we present the results of a study employing CARS spectroscopy to $\mathrm{PC}$ from three different cyanobacteria and in different states of aggregation (monomers and trimers). In contrast to earlier work by Szalontai et al., ${ }^{8}$ significant spectral differences were found for monomers and trimers.

\section{MATERIALS AND METHODS}

The cyanobacteria Westiellopsis prolifica (W.p.) and Spirulina platensis (S.p.) were obtained from the National Facility for Blue-green Algae, Indian Agricultural Research Institute, New Delhi, India. They were grown in BG 11 medium under continuous illumination with fluorescence lamps at $28^{\circ} \mathrm{C}$. Mastigocladus laminosus (M.l.) was grown at the Gesellschaft für Biotechnologische Forschung, Stöckheim, Germany. PC trimers were isolated as described earlier. ${ }^{10-12}$ Monomers of PC were obtained by the addition of the chaotropic agent KSCN (final concentration $1 M) .^{\prime 2}$

Absorption spectra were recorded on a Perkin-Elmer (model Lambda 2) spectrometer before and after each measurement of the CARS spectra. No spectral changes were observed during the CARS measurement, except that in some cases a uniform decrease of less than $5 \%$ was observed for the visible absorption band due either to partial photobleaching or to sedimentation of the biliproteins.

A scheme of our CARS apparatus is given in Fig. 1. Two dye lasers, both pumped by one excimer laser, produce the pump- and Stokes-beam, respectively. These are focused into the cooled $\left(4^{\circ} \mathrm{C}\right)$ sample cuvette under the so-called phase match angle. The CARS 


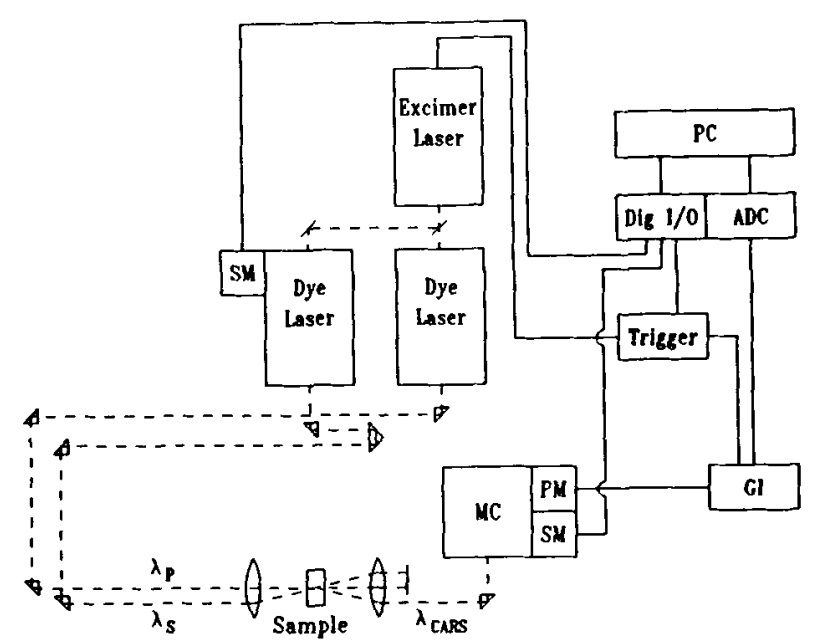

Figure 1. Scheme of the CARS apparatus (ADC: analog-digital converter; Dig I/O; digital input-output; GI: gated integrator; MC: monochromator; PM: photomultiplier; SM: stepping motor).

beam is directed into a double monochromator for separation of stray light and recorded by means of a gated integrator. Each spectrum consists of up to 500 data points, each of which is gained by averaging over 20 laser shots. When recording a CARS spectrum, Stokes laser and double monochromator are scanned synchronously to longer and shorter wavelengths, respectively, by stepping motors. Step width is $0.5 \mathrm{~cm}^{-1}$; it equals the laser band width of approximately $0.5 \mathrm{~cm}^{-1}$. The CARS spectra shown below were recorded with samples of optical densities between $6 / \mathrm{cm}$ and $10 / \mathrm{cm}$ at the long-wavelength absorption maximum. The optical path length in the sample cuvette was $2 \mathrm{~mm}$; laser pulse intensity was $I_{p} \approx 1 \mathrm{~mJ}$, $I_{\mathrm{s}} \approx 0.5 \mathrm{~mJ}$, radius of focus $\approx 0.4 \mathrm{~mm}$, pulse duration $\approx 15 \mathrm{~ns}$, repetition rate $10 \mathrm{~Hz}$. The phase matching conditions were chosen such that most bands appear in the spectra with positive Lorentzian line shape. The solid lines seen in the spectra are the best fits based on the usually applied expression ${ }^{6.7 .13}$ for third-order susceptibility $\chi^{(3)}$ :

$$
\left|\chi^{(3)}\right|^{2}=\left|B+\sum_{r=1}^{n} \frac{R_{r}+i I_{r}}{\Delta_{r}-i \Gamma_{r}}\right|^{2}
$$

where $B$ is nonresonant background; $R_{r}$ and $I_{r}$ are the real and imaginary part of the resonance-enhanced contribution due to the molecular vibration $v_{\mathrm{r}} ; \Gamma_{\mathrm{r}}$ is Raman linewidth of vibration $v_{r} ; \Delta_{\mathrm{r}}$ is $v_{\mathrm{r}}-$ $\left(v_{p}-v_{s}\right)$ with $v_{r}, v_{p}, v_{s}$ wave number of molecular vibration, pumpand Stokes-laser, respectively, and $n$ is number of vibrations that contribute to $\chi^{(3)}$.

\section{RESULTS}

The absorption spectra of PC from the three species are similar but show several species-specific differences (Fig. 2). Upon disaggregation to monomers, a distinct blue shift is observed in W.p. and M.l., but not in S.p. (possibly, this sample is not purely monomeric). These observations indicate that the changes in chromophore geometry and/or protein interaction upon aggregation are different in the three species. However, it is not possible to infer from the absorption spectra which of the three nonequivalent chromophores is responsible for the observed differences or what is their origin (e.g., a change in geometry or excitonic coupling).

Because it has been demonstrated by many authors that both the fingerprint region $\left(1200-1300 \mathrm{~cm}^{-1}\right)$ and the doublebond stretching region (1550-1700 $\left.\mathrm{cm}^{-1}\right)$ are most suitable

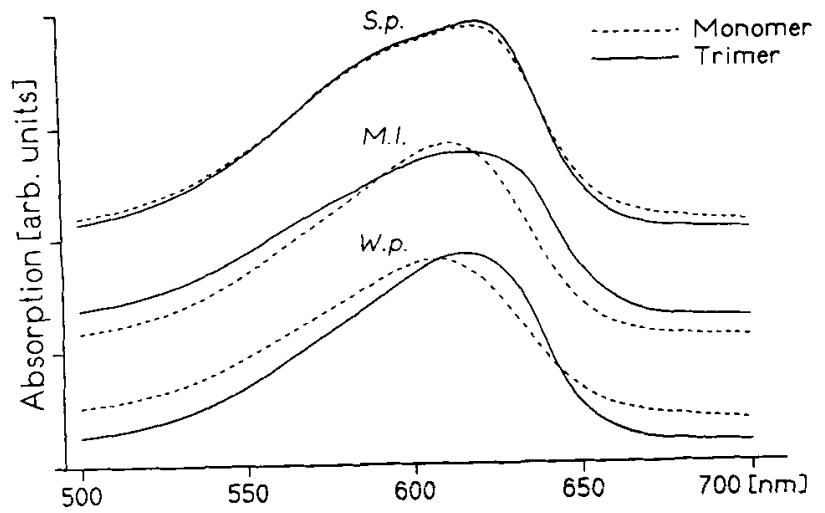

Figure 2. Long-wavelength absorption spectra of phycocyanin trimers (solid line) and monomers (dashed line) from three species of cyanobacteria (Mastigocladus laminosus, Spirulina platensis and Westiellopsis prolifica).

to discuss changes in chromophore structure, the presentation of the CARS spectrum of each species is limited to these regions (Figs. 3-5). The recorded spectra are shown without any data processing, the solid lines represent, as mentioned above, least-squares fits to the experimental curves. The relevant parameters (vibrational frequency $v$, width of the band
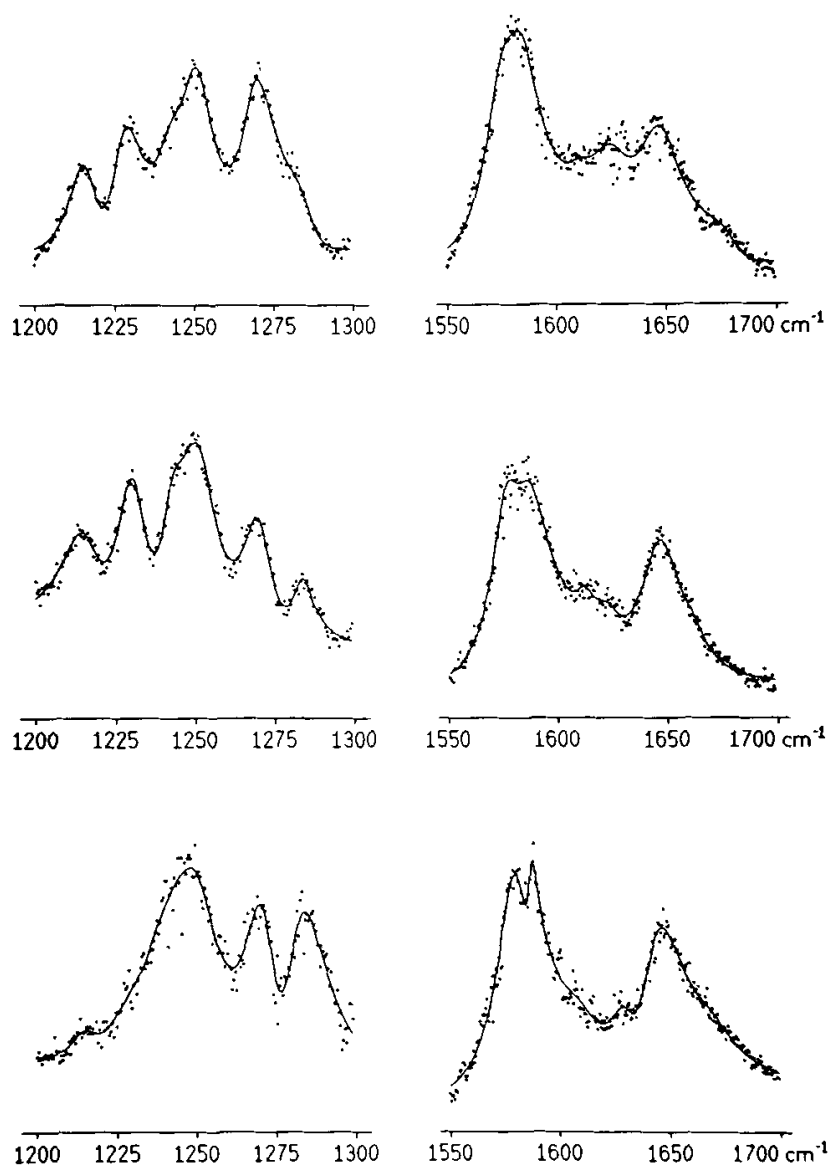

$\begin{array}{lllll}1200 \quad 1225 \quad 1250 & 1275 & 1300\end{array}$

Figure 3. CARS spectra of phycocyanin trimers of Spirulina platensis (top), Mastigocladus laminosus (middle) and Westiellopsis prolifica (bottom) recorded with $\lambda_{\mathrm{p}}=630 \mathrm{~nm}$. 

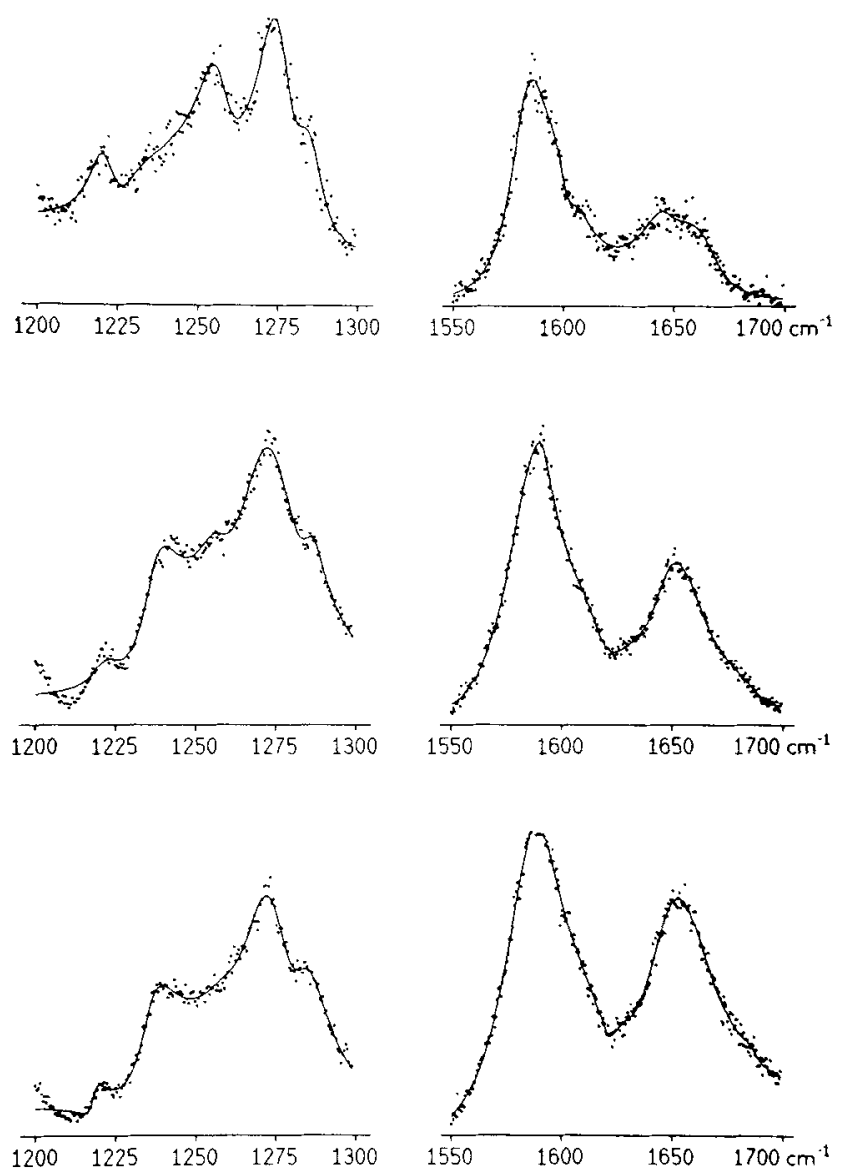

Figure 4. CARS spectra of phycocyanin monomers of Spirulina platensis (top), Mastigocladus laminosus (middle) and Westiellopsis prolifica (bottom) recorded with $\lambda_{\mathrm{p}}=630 \mathrm{~nm}$.

$\Gamma$, relative intensity $\rho$ and $\left.\alpha=\arctan \left[\mathrm{I}_{\mathrm{r}} / \mathrm{R}_{\mathrm{r}}\right]\right)$ are summarized in Tables 1 and 2.

The fingerprint region of the trimers is composed of three to five bands of comparable intensity, whereas in the monomer spectra only three band positions are emphasized by band maxima or pronounced shoulders. In the trimer spectra of S.p. and M.l. the band pattern is almost identical and it is only the band around $1270 \mathrm{~cm}^{-1}$ that is relatively more intense in S.p. In W.p. the bands at 1215 and $1226 \mathrm{~cm}^{-1}$ are extremely weak, and a shoulder at $1237 \mathrm{~cm}^{-1}$ is barely visible. Nevertheless, the band positions found by the fit procedure are very close to those of the other two species (Table 1a). In the monomer spectra the most prominent band is found around $1270 \mathrm{~cm}^{-1}$. The band around $1283 \mathrm{~cm}^{-1}$ is clearly seen in all spectra, but its intensity relative to that around $1275 \mathrm{~cm}^{-1}$ is changed when compared with the trimers. The trimer band found around $1240 \mathrm{~cm}^{-1}$ seems to be missing in the monomer spectra. Whereas the trimer spectra of S.p. and M.l. have close resemblance to each other, the monomer spectra of M.l. and W.p. are nearly identical.

In the double-bond region six bands can be deduced from the fits of both the trimer and the monomer spectra (Table 2). In all three trimer samples a fairly strong band is observed around $1646 \mathrm{~cm}^{-1}$; a similar band is found in the monomer spectra around $1654 \mathrm{~cm}^{-1}$. The most intense feature in all
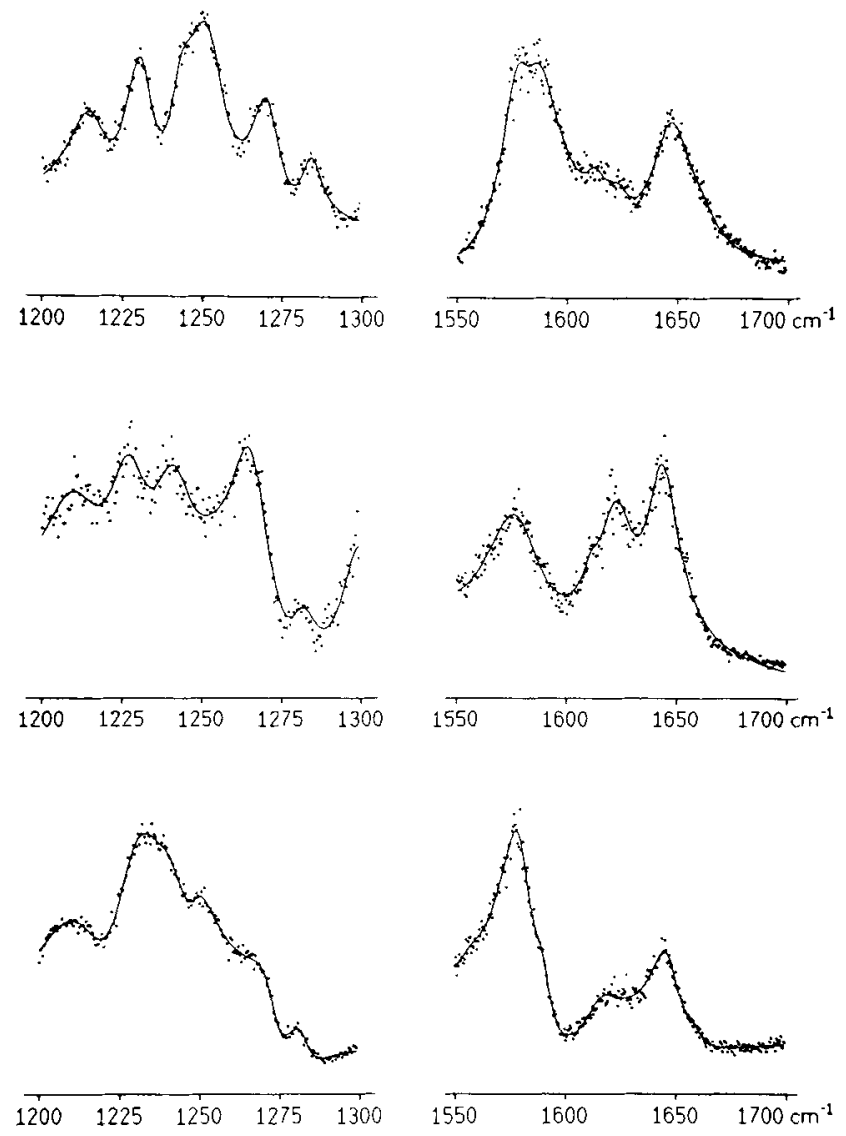

Figure 5. CARS spectra of phycocyanin trimers of Mastigocladus laminosus recorded with three different pump wavelengths: $\lambda_{p}=630$ $\mathrm{nm}$ (top), $\lambda_{\mathrm{p}}=605 \mathrm{~nm}$ (middle) and $\lambda_{\mathrm{p}}=585 \mathrm{~nm}$ (bottom).

spectra is composed of two poorly resolved bands around $1580 \mathrm{~cm}^{-1}$, whose intensity ratio depends on the species. The most intriguing difference between monomer and trimer spectra is, however, that in the monomer some bands in the gap between the band at $1646 \mathrm{~cm}^{-1}$ and the double band around $1580 \mathrm{~cm}^{-1}$ are apparently absent or very weak.

For M.1., CARS spectra were recorded with three different pump wavelengths (Fig. 5). In the fingerprint region six bands can be identified with nearly equal band positions, but strongly different relative intensities (Table $1 \mathrm{~b}$ ). In the double-bond region the remarkable observation is the appearance of a fairly strong band at $1622 \mathrm{~cm}^{-1}$ accompanied by a shoulder at $1612 \mathrm{~cm}^{-1}$ in the spectrum recorded with $\lambda_{\mathrm{p}}=605 \mathrm{~nm}$. In the spectrum recorded with $\lambda_{\mathrm{p}}=585 \mathrm{~nm}$, one medium strong band is seen at $1615 \mathrm{~cm}^{-1}$, whereas in the $630 \mathrm{~nm}$ spectrum the two bands positioned at $1611 \mathrm{~cm}^{-1}$ and 1623 $\mathrm{cm}^{-1}$, respectively, are deduced by the fit procedure from the weak features. The pair of bands found at $1577 / 1584 \mathrm{~cm}^{-1}$ in the $630 \mathrm{~nm}$ spectrum also exhibits an unexpected variation in relative intensity with change of pump wavelength. With $\lambda_{\mathfrak{p}}=605 \mathrm{~nm}$, the band at $1577 \mathrm{~cm}^{-1}$ predominates, whereas with $\lambda_{\mathrm{p}}=585 \mathrm{~nm}$ this band is missing and the $1581 \mathrm{~cm}^{-1}$ band shows comparable intensity to that recorded at $\lambda_{\mathrm{p}}=$ $630 \mathrm{~nm}$ (due to the strongly dispersive line shape of the 1581 $\mathrm{cm}^{-1}$ band, the appearance of the spectrum is misleading at 
Table 1. Fit parameters for the CARS spectra of phycocyanin trimers

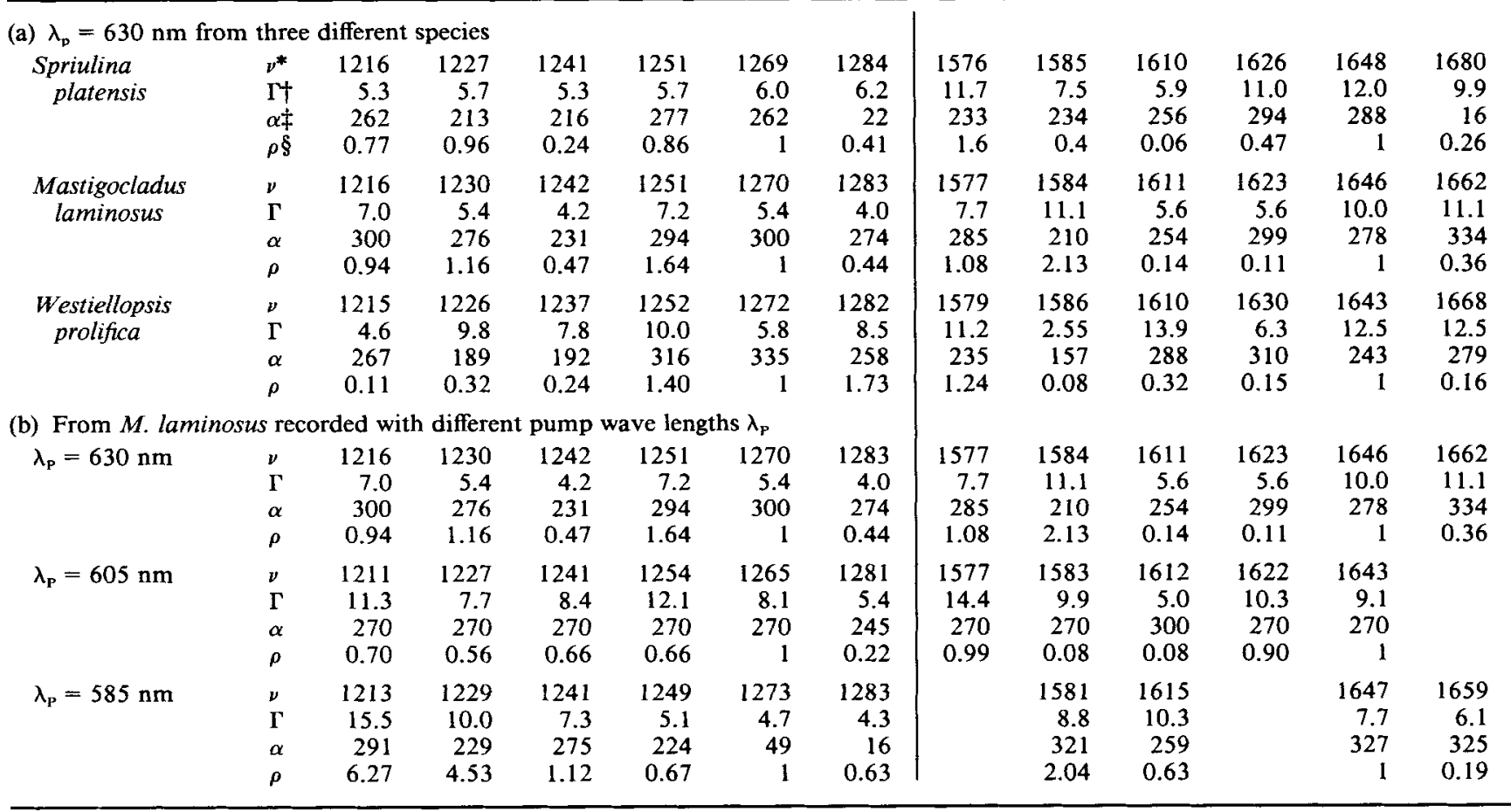

* Frequency in $\mathrm{cm}^{-1}$.

+ Band width in $\mathrm{cm}^{-1}$

$\ddagger$ Arctan $I_{r} / R_{r}$ (see Eq. 1).

$\S$ Relative intensity (normalized separately for fingerprint and double-bond regions).

first glance, because one could assume that the $1577 \mathrm{~cm}^{-1}$ band is still present in the $585 \mathrm{~nm}$ spectrum).

\section{DISCUSSION}

It is obvious from inspection of Figs. 3-5 that the CARS spectra recorded for monomers and trimers, respectively, originating from different organisms, differ to such an extent that one can postulate that the chromophores in PC of dif- ferent organisms must exhibit significant differences in geometry or, at least, must be subject to different interaction with the surrounding protein. It should be emphasized here that the spectra are recorded under identical conditions, e.g., that the optical densities of the samples at the pump wavelength were nearly equal and that the phase match angle was kept constant as well. Spectra taken under these conditions from various aliquots of the same sample prove the good reproducibility of the CARS spectra. The observed spectral differences must therefore be related to distinct differences

Table 2. Fit parameters for the CARS spectra of phycocyanin monomers $\left(\lambda_{\mathrm{p}}=630 \mathrm{~nm}\right.$ ) from three different species (Spirulina platensis, Mastigocladus laminosus and Westiellopsis prolifica)

\begin{tabular}{|c|c|c|c|c|c|c|c|c|c|c|c|c|c|c|}
\hline S. platensis & $\begin{array}{l}\nu^{*} \\
\Gamma \dagger \\
\alpha \neq \frac{\ddagger}{\dot{1}} \\
\rho \S\end{array}$ & $\begin{array}{r}1220 \\
5.3 \\
268 \\
0.28\end{array}$ & $\begin{array}{r}1227 \\
8.2 \\
147 \\
0.29\end{array}$ & $\begin{array}{r}1256 \\
6.0 \\
321 \\
0.39\end{array}$ & $\begin{array}{r}1275 \\
7.0 \\
334 \\
1\end{array}$ & $\begin{array}{r}1284 \\
5.7 \\
290 \\
0.46\end{array}$ & & $\begin{array}{r}1583 \\
10.5 \\
205 \\
1.26\end{array}$ & $\begin{array}{r}1600 \\
6.5 \\
26 \\
0.43\end{array}$ & $\begin{array}{r}1603 \\
9.4 \\
248 \\
0.69\end{array}$ & & $\begin{array}{r}1646 \\
8.8 \\
353 \\
0.28\end{array}$ & $\begin{array}{r}1654 \\
17.1 \\
284 \\
1\end{array}$ & $\begin{array}{r}1667 \\
11.5 \\
39 \\
0.32\end{array}$ \\
\hline M. laminosus & $\begin{array}{l}\nu \\
\Gamma \\
\alpha \\
\rho\end{array}$ & $\begin{array}{r}1222 \\
6.3 \\
231 \\
0.09\end{array}$ & $\begin{array}{r}1236 \\
7.1 \\
196 \\
0.31\end{array}$ & $\begin{array}{r}1256 \\
6.3 \\
295 \\
0.10\end{array}$ & $\begin{array}{r}1272 \\
12.2 \\
276 \\
1\end{array}$ & $\begin{array}{r}1285 \\
4.4 \\
247 \\
0.09\end{array}$ & & $\begin{array}{r}1583 \\
16.4 \\
249 \\
0.80\end{array}$ & $\begin{array}{r}1591 \\
5.6 \\
298 \\
0.04\end{array}$ & & $\begin{array}{r}1618 \\
10.2 \\
117 \\
0.10\end{array}$ & $\begin{array}{r}1641 \\
10.0 \\
250 \\
0.07\end{array}$ & $\begin{array}{r}1652 \\
16.7 \\
102 \\
1\end{array}$ & $\begin{array}{r}1688 \\
8.9 \\
310 \\
0.04\end{array}$ \\
\hline W. prolifica & $\begin{array}{l}\nu \\
\Gamma \\
\alpha \\
\rho\end{array}$ & $\begin{array}{r}1219 \\
2.3 \\
163 \\
0.05\end{array}$ & $\begin{array}{r}1235 \\
7.1 \\
191 \\
0.37\end{array}$ & $\begin{array}{r}1260 \\
6.8 \\
339 \\
0.06\end{array}$ & $\begin{array}{r}1275 \\
9.6 \\
315 \\
1\end{array}$ & $\begin{array}{r}1285 \\
7.6 \\
255 \\
0.46\end{array}$ & $\begin{array}{r}1577 \\
20.2 \\
156 \\
1.02\end{array}$ & $\begin{array}{r}1586 \\
13.3 \\
145 \\
0.74\end{array}$ & & & $\begin{array}{r}1622 \\
5.2 \\
60 \\
0.05\end{array}$ & $\begin{array}{r}1638 \\
14.2 \\
86 \\
0.29\end{array}$ & $\begin{array}{r}1654 \\
19.3 \\
258 \\
1\end{array}$ & $\begin{array}{r}1687 \\
4.0 \\
333 \\
0.01\end{array}$ \\
\hline
\end{tabular}

\footnotetext{
* Frequency in $\mathrm{cm}^{-1}$.

$\uparrow$ Band width in $\mathrm{cm}^{-1}$.

$\ddagger \operatorname{Arctan} I_{r} / R_{r}$ (see Eq. 1).

$\$$ Relative intensity (normalized separately for fingerprint and double-bond regions).
} 
of the samples, and even weak features can be taken as significant information.

One of the advantages of electronic resonance enhancement is the fact that those species, whose absorption is in resonance with the pump wavelength, contribute relatively more to the CARS signal than other species that are not at resonance (the CARS signal is approximately proportional to $\left.\epsilon^{2}\left[\lambda_{p}\right]\right)^{7,34}$ In that sense, one must be aware that differences in the spectra of PC trimers of M.1. when recorded with different pump wavelengths (Fig. 5) are in part due to varying signal contributions of the different chromophores (see below). Because, on the other hand, the pump wavelength $\lambda_{p}$ $=630 \mathrm{~nm}$ falls into the long-wavelength slope of all absorption spectra, one must conclude that the differences observed in the spectra of monomers and trimers of each of the three different species are not caused by different enhancement factors for various chromophores but rather express different chromophore-protein arrangements, especially around chromophore $\beta 84$. Because the resonance-enhanced CARS spectra are, like the spontaneous Raman spectra, governed by the so-called A-term scattering, ${ }^{15}$ the relative intensities of the various bands do reflect the Franck-Condon factors for the various normal coordinates between electronic ground and (first) excited state. If two corresponding bands appear at the same location, but with different relative intensities, then the change in equilibrium distance along this normal coordinate must be different for the two species. Such a situation can be realized if the interaction with the protein, especially the polarization of the $\pi$-electrons in the excited state, is different. A change in the adiabatic wavefunction of the excited electronic state can also be the consequence of excitonic coupling between the locally excited states of close lying chromophores. The latter interpretation would hold especially for the transition from the monomeric to trimeric aggregation state, because the chromophores $\alpha 84$ and $\beta 84$ of different monomers approach each other very closely upon aggregation. ${ }^{4}$

For a better understanding of the CARS spectra, we have performed model calculations using the WARSHEL program for normal coordinate analysis. ${ }^{16}$ Assuming a chromophore geometry as given by the X-ray analysis for M.1.4 and applying the standard force field, i.e., without including interaction with the protein surrounding, the normal mode frequencies are slightly different for each of the three chromophores in accordance with the small differences in molecular geometry. In the region between 1200 and 1300 $\mathrm{cm}^{-1}$ a rather dense sequence of normal modes comprising the NH-bending and methine $\mathrm{CH}$-bending motions are calculated. The normal coordinates in the double-bond stretching region between $1550 \mathrm{~cm}^{-1}$ and $1700 \mathrm{~cm}^{-1}$ should be fairly localized. Around $1680 \mathrm{~cm}^{-1}$ the two carbonyl stretching frequencies as well as the $\mathrm{C}=\mathrm{C}$ stretching of the methine group between ring $\mathrm{A}$ and $\mathrm{B}$ should be found. The $\mathrm{C}=\mathrm{C}$ stretch of ring $D$ should be located around $1625 \mathrm{~cm}^{-1}$, whereas the $\mathrm{C}=\mathrm{C}$ stretch at carbon $\mathrm{C}-10$ is predicted to occur around $1590 \mathrm{~cm}^{-1}$. Pyrrole vibrations of rings $B$ and $C$ should be found between $1550 \mathrm{~cm}^{-1}$ and $1600 \mathrm{~cm}^{-1}$. The exocyclic double-bond stretch between rings $C$ and $D$ is predicted at $1660 \mathrm{~cm}^{-1}$ in the extended chromophore geometry and around $1630 \mathrm{~cm}^{-1}$ in the cyclic helical conformation. It has been suggested that the double-bond region is indicative of the overall chromophore geometry (that is: fully extended, semiextended or cyclic helical), whereas the fingerprint region is indicative for hydrogen bonding between chromophore and protein environment, especially for the strength of the salt bridge between the aspartate residue and the nitrogens of rings $\mathrm{B}$ or $\mathrm{C} .9,17$

The problem that arises in the attempt to make a clearcut statement on the geometry of the three nonequivalent chromophores in the monomeric unit lies in the fact that their absorption spectra overlap strongly. ${ }^{18.19}$ Probably at no pump wavelength lying within the red absorption band, the CARS spectrum is exclusively due to one chromophore only. This is illustrated very well in Fig. 5. Some bands, which are seen in the spectrum recorded with pump wavelength $\lambda_{p}=585$ $\mathrm{nm}$, are weak(er) in the spectrum recorded with pump wavelength $605 \mathrm{~nm}\left(e . g ., 1583\right.$ and $\left.1660 \mathrm{~cm}^{-1}\right)$ and appear again as strong features with pump wavelength $630 \mathrm{~nm}$. One must, therefore, conclude that the spectra of the individual chromophores in their species-dependent native protein environment are much more different than one would expect just by the fact that the analysis of the CARS spectra taken at different pump wavelengths shows the same sets of vibrational frequencies but with different relative amplitudes.

If one assumes that the spectra taken at $630 \mathrm{~nm}$ are dominated by the contributions of chromophore $\beta 84$, those at $605 \mathrm{~nm}$ by $\alpha 84$ and those at $585 \mathrm{~nm}$ by chromophore $\beta 155$, then the following conclusions can be drawn:

(1) The band around $1645 \mathrm{~cm}^{-1}$, which is observed for each pump wavelength (Fig. 5), confirms that in M.1. (in solution) all three chromophores are stabilized in the protein in the fully extended conformation. It has been found experimentally that in samples, in which the chromophore exhibits an extended conformation, a band is observed between 1645 and $1655 \mathrm{~cm}^{-1}$. Upon denaturation of such samples, ${ }^{8}$ that is upon transition from the extended to the cyclic helical conformation, this band shifts to about $1620 \mathrm{~cm}^{-1}$ in accordance with theoretical predictions. An extended conformation is verified also for the $\beta 84$ chromophore in both monomers and trimers isolated from the three different species (spectra of Figs. 3 and 4).

(2) If the bands at 1622 and $1612 \mathrm{~cm}^{-1}$ are characteristic for the $\alpha 84$ chromophore, then this chromophore must be subject to changes upon aggregation from the monomeric to the trimeric state (filling-up of the gap). This conclusion is also supported by the following observation: In the case of S.p. and M.1., for which the spectral changes upon aggregation in the double bond region around $1620 \mathrm{~cm}^{-1}$ are most pronounced, one also finds the most drastic changes in the fingerprint region between 1200 and $1250 \mathrm{~cm}^{-1}$. It has been observed earlier, that only the $\alpha$-subunit shows bands in this spectral region, whereas the $\beta$ - subunit $^{14}$ exhibits vibrational bands only above $1250 \mathrm{~cm}^{-1}$. The filling-up of the gap around $1620 \mathrm{~cm}^{-1}$ is therefore in accordance with the appearance of the two bands around 1215 and $1230 \mathrm{~cm}^{-1}$. The changes of the $\alpha$-chromophore appear, however, to be species dependent; the electronic structure in the W.p. trimer is closer to that found in the monomers than in S.p. and M.L.

(3) In the trimer spectra of M.1. recorded with $\lambda_{p}=585$ $\mathrm{nm}$ and $\lambda_{\mathrm{p}}=630 \mathrm{~nm}$, a strong band around $1580 \mathrm{~cm}^{-1}$ is observed. This indicates that both chromophores $\beta 84$ and $\beta 155$ but not $\alpha 84$ must possess a vibration around $1580 \mathrm{~cm}^{-1}$. 
Because in the $\lambda_{\mathrm{p}}=630 \mathrm{~nm}$ spectra of all three species, this band is changed upon aggregation, one must conclude that most likely chromophore $\beta 84$ also changes its electronic structure upon aggregation.

(4) Based on the above tentative assignment, according to which the bands between 1550 and $1600 \mathrm{~cm}^{-1}$ are related to vibrations of rings $\mathrm{B}$ and $\mathrm{C}$ and the methine bridge $\mathrm{C}-10$, the observed spectral changes upon aggregation must be interpreted as being due to changes of the specific interaction of these two rings with the surrounding protein.

(5) From the X-ray data it is suggested that $\beta 84$ and $\beta 155$ experience different chromophore-protein interactions in the region of the ring $\mathrm{B}, \mathrm{C}$ fragment. Possibly, this difference is reflected in the different intensity distribution in the fingerprint region (especially in the intensity ratio of the bands between 1250 and $1300 \mathrm{~cm}^{-1}$ ), when the pump wavelength is changed from 630 to $585 \mathrm{~nm}$.

\section{CONCLUDING REMARKS}

In the UV-excited resonance-enhanced Raman spectra of $\mathrm{C}-\mathrm{PC}^{8}$ only one strong band and one or two weak features could be observed in each of the two spectral regions discussed above (1200-1300 $\mathrm{cm}^{-1}$ and $\left.1550-1700 \mathrm{~cm}^{-1}\right)$. Furthermore, no significant changes were found upon transition in the state of aggregation from trimeric to monomeric by addition of acid. It is obvious that in these experiments, the recorded spectrum is the unresolved superposition of the spectra of different types of chromophore-protein arrangements. Besides the differentiation according to the binding site $(\alpha 84, \beta 84, \beta 155)$, a second level of heterogeneity (microheterogeneity) exists, because these three types of chromophores can experience different protein environments, which in turn could cause different geometric and electronic structures of the chromophores. All these heterogeneities are reflected in the pump-wavelength dependence of the CARS spectra, in which about six bands can be identified beyond doubt in each of the two spectral regions. In view of the theoretical results, it is likely that bands which show opposite trends in intensity upon a change of excitation wavelength belong to different types of chromophores $(\alpha 84, \beta 84, \beta 155)$. Additionally, one can postulate that the small variations in vibrational frequency caused by differences in the protein environment and small variations in chromophore geometry are responsible for the increased width of several bands. In any case, one can conclude from the differences observed in the CARS spectra of the investigated PC that there must be changes in the electronic structure of the various chromophores upon aggregation from the monomeric to the trimeric state.

Significant spectral changes have also been observed upon aggregation of allophycocyanin, ${ }^{20}$ or between allophycocyanin trimers with and without linkers, and upon denaturation. The frequency shifts can be due to changes in molecular geometry, to changes in the force constants, which are influenced $e . g$. by coulombic interaction with the protein, but also by variations in the excited state properties (excitonic coupling). At the present time, it is not possible to decide between these possibilities. One can hope that with more experimental material being available and by making use of the results of molecular modeling and normal mode calculations, more specific conclusions can be drawn about the differences in chromophore-protein arrangement.

Acknowledgement-Financial support by Fonds der Chemie and Deutsche Forschungsgemeinschaft (SFB 143) is gratefully acknowledged.

\section{REFERENCES}

1. Gantt, E., C. A. Lipschultz, J. Grabowski and B. K. Zimmerman (1979) Phycobilisomes from blue-green and red algae: isolation criteria and dissociation characteristics. Plant Physiol. 63, 615620.

2. Scheer, H. (1986) Excitation transfer in phycobiliproteins. In Encyclopedia of Plant Physiology, Vol 19. Photosynthesis III (Edited by L. A. Staehelin and C. J. Arntzen), pp. 327-337. Springer-Verlag, Berlin.

3. MacColl, R. and D. Guard-Friar (1987) Phycobiliproteins. CRC Press, Boca Raton, FL.

4. Schirmer, T., W. Bode and R. Huber (1987) Refined threedimensional structures of two cyanobacterial C-phycocyanins at 2.1 and $2.5 \AA$ resolution. J. Mol. Biol. 196, 677-695.

5. Scharnagl, C. and S. Schneider (1991) UV-visible absorption and circular dichroism spectra of the subunits of C-phycocyanin II: a quantitative discussion of the chromophore-protein and chromophore-chromophore interactions in the $\beta$-subunit. $J$. Photochem. Photobiol. 8, 129-157.

6. Schneider, S., F. Baumann, U. Klüter and P. Gege (1988) Resonance-enhanced CARS spectroscopy of biliproteins. Croat. Chem. Acta 61, 505-527.

7. Schneider, S., F. Baumann and P. Gege (1988) Resonance CARS spectra of allophycocyanin. In Proceedings of the XIth International Conference on Raman Spectroscopy (Edited by $\mathbf{R}$. J. H. Clark and D. A. Long), pp. 639-640. John Wiley \& Sons, London.

8. Szalontai, B., Z. Gombos, V. Csizmedia and M. Lutz (1987) The chromophore structure and chromophore-protein interactions in $C$-phycocyanin as studied by resonance Raman spectroscopy. Biochem. Biophys. Acta 893, 296-304.

9. Szalontai, B., Z. Gombos, V. Csizmadia, K. Csatorday and M. Lutz (1989) Chromophore states in allophycocyanin and phycocyanin Biochem 28, 6467-6472.

10. Menon, V. K. N., A. Kumar, A. L. Menon and A. K. Varma (1988) A rapid procedure for isolating phycobilisomes from cyanobacteria. Anal. Biochem. 172, 377-381.

11. Maruthi Sai, P. S. (1990) Characterisation of light-harvesting pigment complexes in cyanobacteria at a molecular level. Ph.D. Thesis, Jawaharlal Nehru University, New Delhi, India.

12. Fischer, R., J. Gottstein, H. Scheer, P. Geiselhart and S. Schneider (1990) Picosecond time-resolved fluorescence of phycobiliproteins: subunits of phycocyanin from Mastigocladus laminosus. J. Photochem. Photobiol. 5, 151-165.

13. Carreira, L. A., L. P. Goss, and T. B. Malloy, Jr. (1981) Applications of CARS to condensed phase systems. In Chemical Applications of Raman Spectroscopy (Edited by A. B. Harvey), pp. 321-376. Academic Press, New York.

14. Baumann, F. (1990) Resonanz-CARS-Spectroskopie an Biliproteiden. Ph.D. Thesis, Techn. Universität München, Germany.

15. Albrecht A. C. (1961) On the theory of Raman intensities. $J$. Chem. Phys. 34, 1476-1484.

16. Warshel A. and M. Levitt (1973) QCPE-program 247, revised version.

17. Debreczeny, M., Z. Gombos., V. Csizmadia, Zs. Varkonyi and B. Szalontai (1989) Chromophore conformational analysis in phycocyanin and in related chromopeptides by surface enhanced Raman spectroscopy. Biochem. Biophys. Res. Commun. 159/3, $1227-1232$

18. Sauer, K., H. Scheer and P. Sauer (1987) Förster transfer calculations based on crystal structure data from Agmenellum quadruplicatum C-phycocyanin. Photochem. Photobiol. 46, 427440.

19. Fischer, R., S. Siebzehnrübl and H. Scheer (1988) C-phyco- 
cyanin from Mastigocladus laminosus: chromophore assignments in higher aggregates by cystein modification. In Photosynthetic Light-Harvesting Systems (Edited by $\mathrm{H}$. Scheer and S. Schneider), pp. 71-76. W. de Gruyter, Berlin.

20. Prenzel, C.-J., G. Brehm, P. Gedeck, S. Schneider and H. Scheer (1992) Effect of aggregation on chromophore structure in al- lophycocyanin studied by resonance CARS-spectroscopy. In Proceedings of the XIIIth International Conference on Raman Spectroscopy (Edited by W. H. Kiefer, M. Cardona, G. Schaack, F. W. Schneider and H. W. Schrötter), pp. 536-537. John Wiley \& Sons, London. 